\title{
Performance of Light Fidelity and Wireless Fidelity Networks in a WLAN
}

\author{
S.N. Arinze, G.N. Onoh and D.O. Abonyi
}

Wireless Fidelity utilizes radio waves. Radio waves have limited bandwidth, fully exploited, and low speed. Light fidelity is a visible light communication used as a medium to carry high-speed data. It can complement the wireless fidelity in accessing the internet, thereby reducing congestion. In this work, a hybrid of light fidelity and wireless fidelity was carried out in an indoor environment. The geometry of the light fidelity access point and wireless fidelity access point was analyzed in the MatLab environment to know the highest power received by the user. Some ranges of received power considered for good reception wereassigned for each of the wireless access points in the MatLab program. Visual Basic, an object-oriented computer programming language, reads the signal coming from the communication port of light fidelity and wireless fidelity. It mimics the analyzed MatLab program of a hybrid network of light fidelity and wireless fidelity and connects to any of the wireless access points based on the set conditions of the satisfied received power. With these, the light fidelity can complement the limited available radio frequency.

\section{Index Terms - Light Fidelity, MatLab, Visual Basic, Wireless Fidelity.}

\section{INTRODUCTION}

Wireless Fidelity network allows more than one device to communicate and share information within defined regions like office, home, and campus. It offers users many benefits, such as portability and flexibility. At anytime, anywhere, network access to resources is obtainable. Users can move around within a local coverage area while still connected to the network. Handheld devices such as smart phones, laptops, and personal digital assistants allow users to synchronize individual databases and provide access to network services. When many devices are connected, clogged airwaves find it challenging to catch a good signal. Many accessories such as microwave ovens, cordless phones, and Bluetoothoperating in similar frequency $(2.4 \mathrm{GHz})$ with the wireless local area network can interfere with the overall network performance. The interference causes the access point to hold off the transmission. The resulting decrease in the network performance can make downloading files to be sluggish, limit the number of active users, and make the wireless clients not to access the wireless local area network for an indefinite time. The effective throughput is often worse than expected, given that the workload of the varying number of users accessing the wireless network cannot be predicted and may not meet the bandwidth. Many users tend to abandon a page if it has not loaded, thereby making them unable to download relevant documents. Many hours can be lost, waiting for pages to load. Some users get the dreaded error that indicates you could not reach the webpage or you have no internet.

S.N.Arinze, G.N. Onoh and D.O. Abonyi are with Electrical and Electronic Engineering Department, Enugu State University of Science and Technology (e-mail:arinze.ndidi@esut.edu.ng,greg.nwachukwu@esut.ng.edu, abonyi.dorathy@esut.ng.edu). 
It makes people lose trust when doing an online transaction. When bandwidth is strained, and connectivity is limited, one cannot work to full potential. These make businesses to dwindle for a while. Productivity and revenue become a problem in the country since people depend on digital technology. However, the loss of confidentiality, integrity, speed, and a large number of users are risks associated with wireless local area network. Unauthorized users may access the system using a Wi-Fi receiver and software. They may corrupt the information, launch attacks to authorized users, prevent them from accessing the network, consume network bandwidth, and degrade network performance. Wireless local area network security is better than nothing since a dedicated hacker can still steal the information[1]. The speed of the wireless local area network is slow when transferring large files and becomes worse when the system is busy, or signal strength is weaker than optimal. The speed is also affected when more devices connected to the wireless fidelity network are actively downloading data at the same time. It is because the bandwidth has to be shared.

Based on this scenario, one could ask if the world can use other frequencies to complement the existing radio frequencies in accessing the internet. A possible approach and adequate answer is light fidelity. Light Fidelity is a visible light communication that has frequencies between $400 \mathrm{THz}(780 \mathrm{~nm})$ to $800 \mathrm{THz}(375 \mathrm{~nm})$. The light spectrum is presently unlicensed, has wide bandwidth, and can exploit existing infrastructures[2]. Light is a common carrier of data as seen in fiber optical network, although it was not so common to send information through the air using visible light. Light has been used to transfer data. For example, infrared light is used in the remote control to transmit a short signal to the television.

\section{THEORY}

Wireless Fidelity (Wi-Fi) is a radio technology governed by the Wi-Fi alliance and used for wireless local area networks. It is a high-speed internet connection that works at a frequency of $2.4 \mathrm{Ghz}$ or $5 \mathrm{Ghz}$. Wi-Fi cards in computers or mobile phones read the signals transmitted from antennas and routers, creates an internet connection between the user and network. It's a two-way communication that works over a long distance than infrared and Bluetooth. Wi-Fi has several standards with different speeds, frequency, and power requirements. 801.11b was its earliest standard. It is the slowest but the least expensive. As time goes on, it upgraded to 802.11a,802.11g, and 802.11n standards[3]. These standards allow longer ranges and data throughput. Wi-Fi has advantages of deployment without cabling, mobility, convenience, expandability, and cost. Despite its benefits, it has drawbacks, which are a decrease in performance as the range increases, interference, vulnerable to attacks, and security issues.

Light Fidelity ( $\mathrm{Li}-\mathrm{Fi})$ is a wireless communication that transmits data using light. It is a visible light communication that sends data using a light-emitting diode (LED) that turns on and off at an incredibly high speed, receives the light signals with a photodiode and processes the data at high speed[4].It is a new technology that was introduced by Harald Hass in the Technology, Entertainment, and Design Global talk on visible light communications. He illustrated the concept of Li-Fi by transmitting a video of a blooming flower using a table lamp with an LED bulb and projected it onto a screen. He blocked the light from the LED bulb and transmission stooped, showing that light can be used to transmit data[5]. Li-Fi uses an electromagnetic spectrum that is still not greatly utilized and can change how the users access the internet, receive emails, stream videos. Li-Fi has an advantage of better security, large throughput, less interference, and large capacity 


\section{MATERIALS AND METHOD}

Assuming a Li-Fi LED bulb and a Wi-Fi access point kept at the ceiling was enabled in an empty room with no obstacles as shown in fig 1

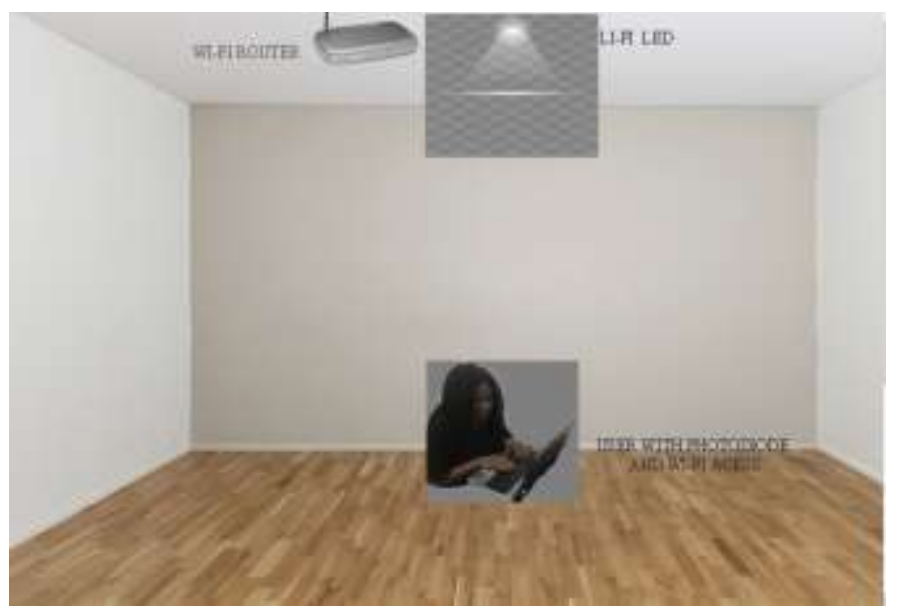

Fig.1 Indoor application of light fidelity and wireless fidelity

The Li-Fi channel was made up of the line of sight (LOS) and non-line of sight (NLOS) paths. The geometry of the indoor Li-Fi is in fig.2. Assuming the user device is fitted with a photodiode vertically facing upwards for simplicity.

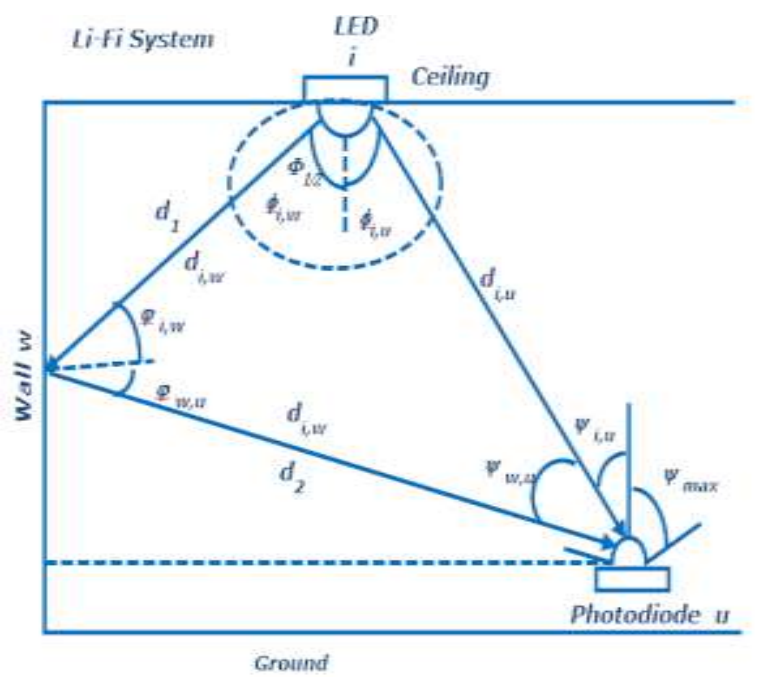

Fig. 2 The geometry of light fidelity path

For the LOS path, the photodiode has a concentrator for focusing of light, filter for passing signals at a specific range of frequency and surface area. The optical gain [6] of the concentrator is

$$
g(\varphi)=\left\lfloor\begin{array}{rl}
\frac{\mathrm{n}^{2}}{\sin ^{2} \varphi_{c}}, & 0 \leq \varphi \leq \varphi_{c} \\
0, & \varphi>\varphi_{c}
\end{array}\right\rfloor
$$

$\mathrm{n}$ is the refractive index of the concentrator, $\varphi$ is the receiver angle and $\varphi_{\mathrm{c}}$ is the concentrator's maximum angle of view. Filter factor is $T_{s}(\varphi)$ and the effective area is 


$$
A_{\text {eff }}(\varphi)=\left[\begin{array}{cc}
A_{r} \cos \varphi, & 0 \leq \varphi \leq \pi / 2 \\
0, & \varphi>\pi / 2
\end{array}\right]
$$

Where $A_{r}$ is the surface area of the photodiode. In adding the optical gain and filter factor, the effective area becomes

$$
\mathrm{A}_{\text {eff }}(\varphi)=\left[\begin{array}{cl}
\mathrm{A}_{\mathrm{r}} \cos \varphi T_{s}(\varphi) \mathrm{g}(\varphi), & 0 \leq \varphi \leq \pi / 2 \\
0, & \varphi>\pi / 2
\end{array}\right]
$$

The optical received power is

$$
P_{r}=H_{d 1}(t) P_{t}
$$

$H_{d}(t)$ is the channel gain and $P_{t}$ is the transmitted power

But $P_{t=} \frac{I(\theta)}{R(\theta)}(5)$

Radiant Lambertian $R(\theta)=\frac{m+1}{2 \pi} \cos ^{m}(\theta)(6)$

Lambertian states that the radiant intensity is directly proportional to the cosine of the angle between the direction of incident light and the surface normal[6].

$m=\frac{-\ln \frac{1}{2}}{\ln \left(\cos \left(\Phi \frac{1}{2}\right)\right)}$ is the Lambertian emission and $\theta$ is the irradiance angle of the LED. $\Phi \frac{1}{2}$ is semi-angle half power and it is the frequency at which the output power has dropped to half of its peak value.

Substituting (6) into (5) gives

$$
\begin{aligned}
& I(\theta)=P_{t} \frac{m+1}{2 \pi} \cos ^{m}(\theta) \\
& \quad \text { And } \\
& P_{r}=I_{r}(\theta) A_{e f f}(\varphi)
\end{aligned}
$$

The intensity of light received has a dividing factor of the quadratic of the distance $d^{-2}$ of the intensity transmitted by LED.

$$
P_{r}=\frac{I(\theta)}{d^{2}} A_{e f f}(\varphi)
$$

Substituting (3) and (7) into (9) gives

$$
P_{r}=P_{t} \frac{m+1}{2 \pi d^{2}} \cos ^{m}(\theta) \mathrm{A}_{\mathrm{r}} \cos \varphi T_{s}(\varphi) \mathrm{g}(\varphi)
$$

Recall from (4) that

$$
P_{r}=H_{d 1}(t) P_{t}
$$

Hence $H_{d 1}(t)=\frac{m+1}{2 \pi d^{2}} \cos ^{m}(\theta) \mathrm{A}_{\mathrm{r}} \cos \varphi T_{s}(\varphi) \mathrm{g}(\varphi)$

The algorithm written in MatLab considered an empty room with xyz dimension, fixed position of the LED on the ceiling, a fixed distance of LED to the photodiode, the effective surface area of the photodiode and optical filter index ' $n$ ' of 1 on the photodiode. The semiangle at half-power was varied using $30^{\circ}, 45^{\circ}$ and $60^{\circ}$ with an LED input power of 10 watts. Considering the NLOS path with only one reflection, as shown in fig. 2 , the reflection has two segments which are from the Li-Fi LED to the small area on the wall and from the wall to the user.

Its channel gain becomes:

$$
H_{d 2}(t)=\left\lfloor\frac{m+1}{2 \pi d 1^{2} d 2^{2}} \cos ^{m}(\theta) \rho \mathrm{d}_{\text {Awall }} \cos \alpha \cos \beta \cos \varphi T_{s}(\varphi) g(\varphi)\right\rfloor
$$

where $d 1$ is the distance between the LED and the reflection point on the wall, $d 2$ is the distance between the reflection point on the wall and the receiver, $\rho$ is the reflection coefficient, $d_{\text {Awall }}$ is the emission area of micro surface, $\alpha\left(\vartheta_{i, w}\right)$ is the radiation angle of the 
reflection of the wall, $\beta\left(\vartheta_{w, u}\right)$ is the radiation angle of the receiver. According to International lightning standard (EN12464-1), the reflection coefficient for light planning in an empty indoor room are:

$0.6 \leq \rho \leq 0.9$ for the ceiling

$0.3 \leq \rho \leq 0.8$ for the wall

$0.1 \leq \rho \leq 0.5$ for the floor

The emission area of the microsurface is $0.00001 \mathrm{~m}^{2}$ [7].

The total Channel gain for both LOS and NLOS is:

$$
H_{d}(t)=H_{d 1}(t)+H_{d 2}(t)
$$

The total received power for both LOS and NLOS is:

$$
P_{\text {rtotal }}=H_{d}(t) P_{t}
$$

The parameters for LOS and NLOS used in the MatLab are in table 1.

Table 1 LOS and NLOS Li-Fi Parameters

\begin{tabular}{ll}
\hline Parameters & Value \\
\hline Room dimension & $\mathrm{x}=3.5, \mathrm{y}=3.5, \mathrm{z}=2.5$ \\
Transmitting Power & 50 Watts \\
Maximum angle field of View & $75^{\circ}$ \\
LED position height (fixed at the ceiling) & $1 \mathrm{x}=0, \mathrm{ly}=0, \mathrm{lz}=0$ \\
Height of Photodiode to the LED & $1.5 \mathrm{~m}$ \\
Half power angle & $30^{\circ}, 45^{\circ}, 60^{\circ}$ \\
Concentrator index (n) & 1 \\
Filter factor & 1 \\
The surface area of the photodiode & $0.0001 \mathrm{~m}^{2}$ \\
Reflection coefficient & 0.6 \\
Emission area of Wall & $0.00001 \mathrm{~m}^{2}$ \\
\hline
\end{tabular}

The geometry of indoor $\mathrm{Wi}-\mathrm{Fi}$ is in fig 3. The received power from the Wi-Fi access point was calculated using the two ray ground reflected model that predicts the path losses between a transmitter and receiver.

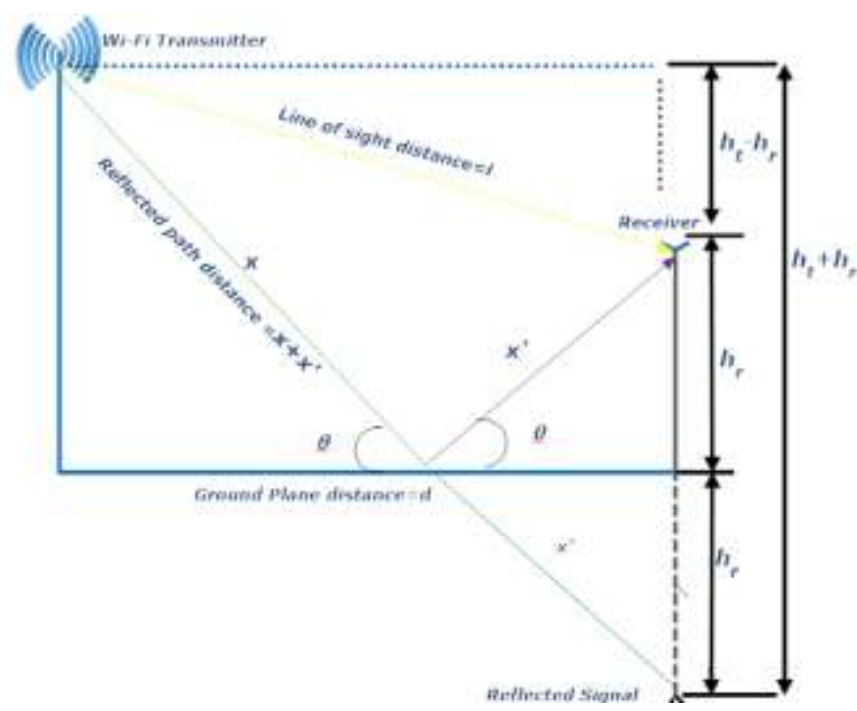

Fig 3. The geometry of the wireless fidelity path 
From fig. 3 the direct line of sight distance is

$$
l=\sqrt{\left(h_{t}-h_{r}\right)^{2}+d^{2}}
$$

and the reflected path distance is

$$
x+x^{\prime}=\sqrt{\left(h_{t}+h_{r}\right)^{2}+d^{2}}
$$

The path length difference is

$$
\Delta d=\sqrt{\left(h_{t}+h_{r}\right)^{2}+d^{2}}-\sqrt{\left(h_{t}-h_{r}\right)^{2}+d^{2}}
$$

The phase difference is

$$
\Delta \emptyset=\frac{2 \pi \Delta d}{\lambda}
$$

$\lambda$ is the wavelength of the transmission

The received line of sight is

$R_{\text {los }}=\sqrt{\frac{G_{\text {los }}}{l}}(18)$

While the received ground reflection is

$$
R_{g r}=R \sqrt{G_{g r}} \frac{e^{j \Delta \emptyset}}{x+x^{\prime}}
$$

where $G_{l o s}$ is the antenna gain along the LOS path, $G_{g r}$ is the antenna gain along the ground reflected path, $\mathrm{R}$ is the reflection coefficient.

The LOS reflection coefficient is ' 1 ' because the signal experiences no loss due to obstructions while the ground reflection coefficient is ' -1 ' because the signal reflects off the ground [8][9].

The received power is

$$
P_{r}=\frac{\lambda\left(R_{\text {los }}+R_{g r}\right)^{2}}{4}
$$

Wi-Fi channel parameters used in the MATLAB code is in table 2.

Table 2 Parameters of Wi-Fi Channel

\begin{tabular}{ll}
\hline Parameters & Value \\
\hline Room dimension & $\mathrm{x}=3.5, \mathrm{y}=3.5, \mathrm{z}=2.5$ \\
Height of transmitter to the ground & $3.5 \mathrm{~m}$ \\
Height of receiver to the ground & $1.5 \mathrm{~m}$ \\
LOS Antenna Gain & 1 \\
Ground reflected Antenna Gain & 1 \\
Frequency & $2.4 \mathrm{GHz}$ \\
Reflection Coeffiecient & -1 \\
\hline
\end{tabular}

Having calculated the received power for both the $\mathrm{Li}-\mathrm{Fi}$ and $\mathrm{Wi}-\mathrm{Fi}$, the integration of both systems is through a written program in MATLAB.

In the program, Li-Fi LED connects to the Arduino board in demo mode. When both Li-Fi and Wi-Fi were enabled, the program calculates the received power of photodiode, and if it is 
not within the specified range assigned for it, it dims the LED and calculates the received power of $\mathrm{Wi}-\mathrm{Fi}$. If the received power of $\mathrm{Wi}-\mathrm{Fi}$ is not within the range, it brightens the LED. The process of dimming and brightening minimizes the energy consumption of the hybrid system. The implementation of it works as application software in the laptop as shown in the flow chart of fig. 4

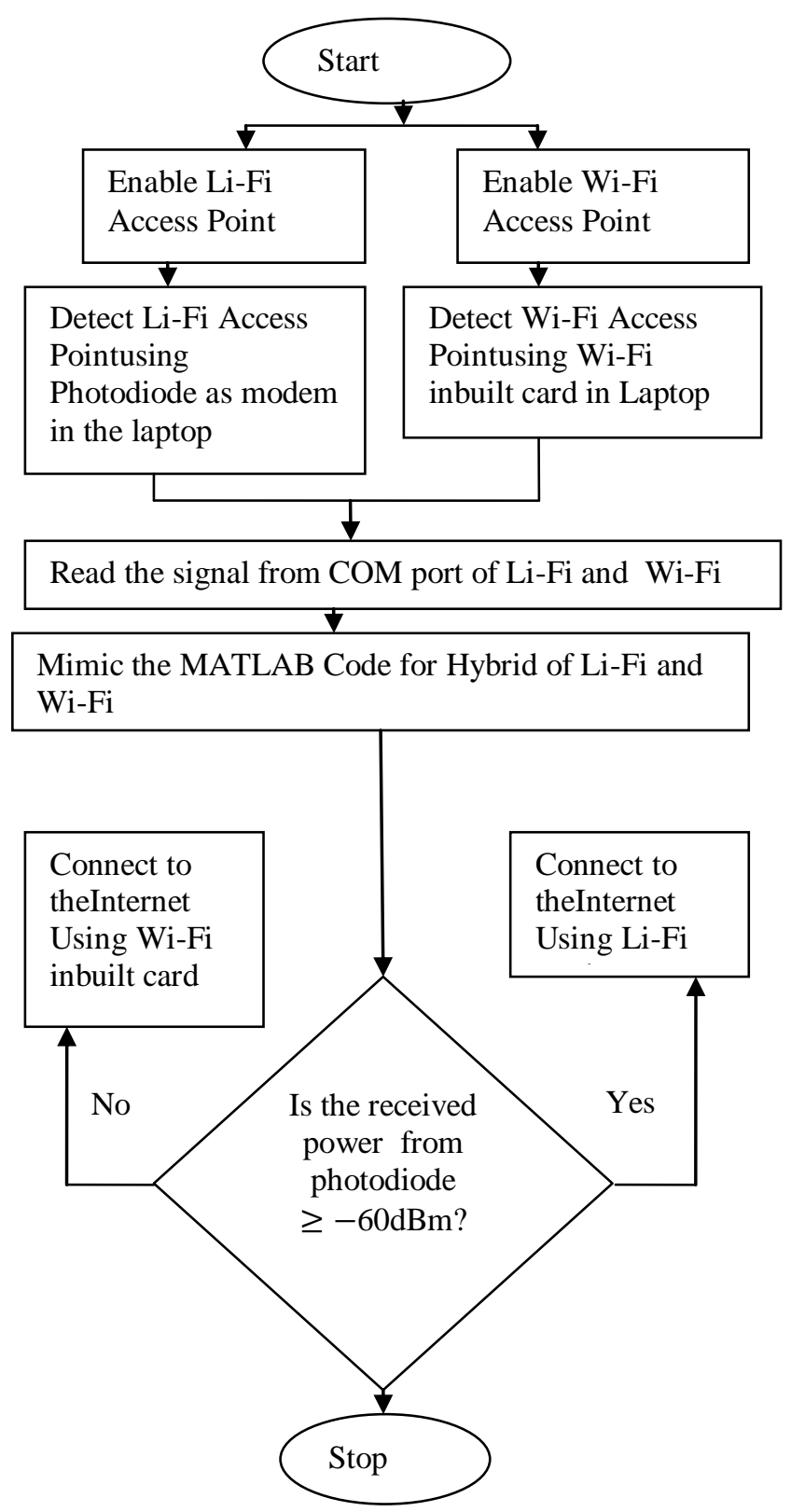

Fig. 4 Implementation process flow chart of Li-Fi and Wi-Fi system

When the laptop device starts, the Li-Fi modem and inbuilt Wi-Fi card in the laptop were enabled. Visual Basic is an object-oriented computer programming language. It read the signal coming from the communication port of $\mathrm{Li}-\mathrm{Fi}$ and $\mathrm{Wi}-\mathrm{Fi}$. It mimics the MATLAB code for the hybrid network and checks if the received power from the photodiode is greater than or equal to $-60 \mathrm{dBm}$ (as specified for $\mathrm{Li}-\mathrm{Fi}$ ). If it satisfies the condition of greater or equal to $-60 \mathrm{dBm}$, it connects to the internet using a Li-Fi modem, but if not, it relates to the internet using $\mathrm{Wi}-\mathrm{Fi}$ inbuilt card on the laptop. 


\section{RESULTS}

The result obtained in varying the semi-angle at half-power of LED from $30^{\circ}, 40^{\circ}$ and $60^{\circ}$ with an input power of 10 watts for the indoor line of sight of $\mathrm{Li}-\mathrm{Fi}$ is in figs 5,6 and 7 respectively.

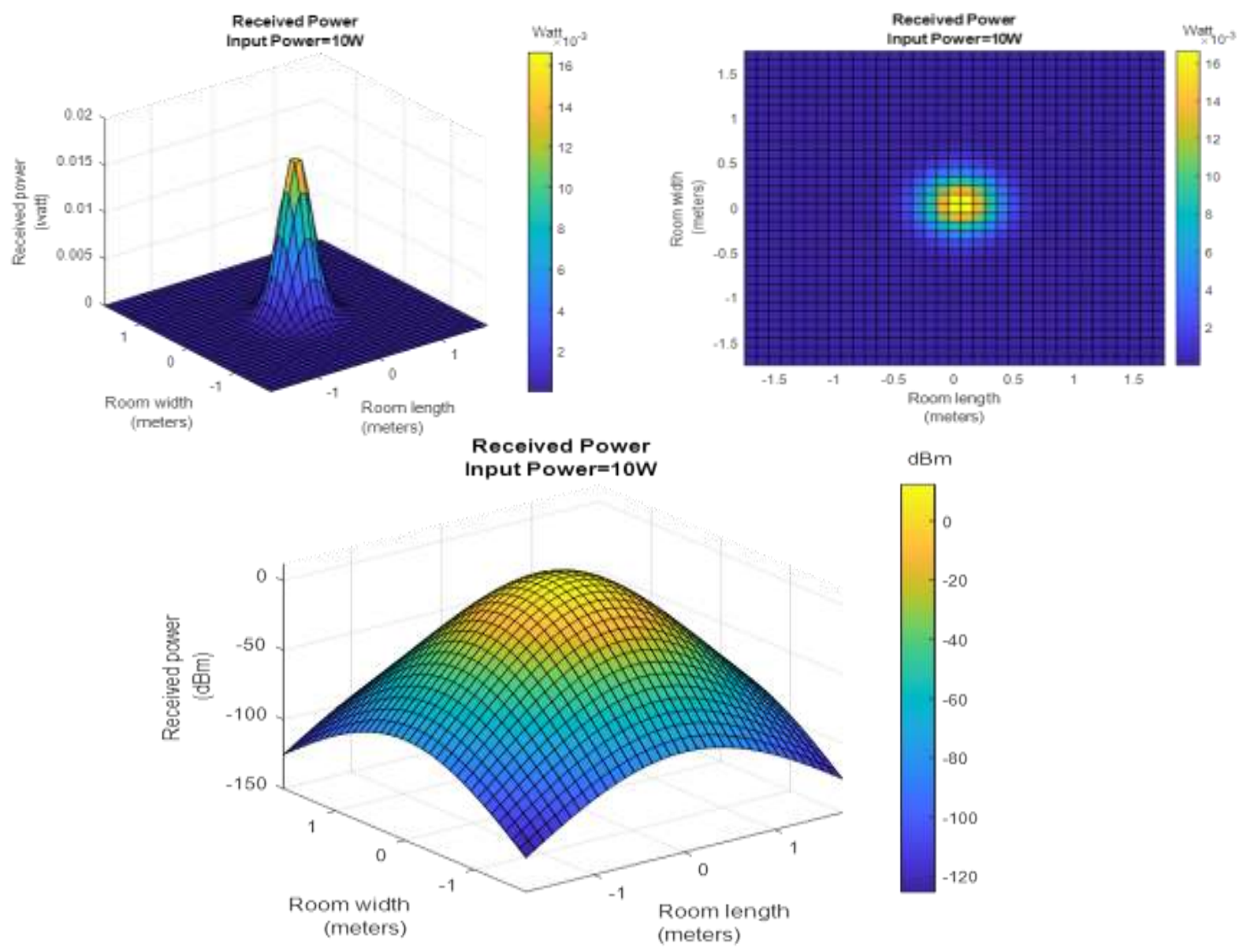

Fig. 5 Received power from photodiode at a half-power angle of $30^{\circ}$

Full yellow color in fig. 5 is the highest power received by the photodiode while the dark blue indicates the lowest power received. The concentrated received power showed that the signal would be directional. The concentrated received power from ranges of $-60 \mathrm{dBm}$ to $0 \mathrm{dBm}$ was considered for good reception of $\mathrm{Li}-\mathrm{Fi}$ while the areas marked dark blue will experience low reception of light.

The received power from the photodiode was diffused in fig. 6 and reduced than the power obtained when the half-power angle was at $30^{\circ}$. The highest received power for good reception is from $-6 \mathrm{dBm}$ to $2 \mathrm{dBm}$.

At half power angle of $60^{\circ}$ in fig 7 , the received power becomes more diffused and less than the received power obtained for the $30^{\circ}$ and $45^{\circ}$. The values for good reception are within the range of $-7 \mathrm{dBm}$ to $-3 \mathrm{dBm}$.

From the analysis, the smaller the half-power angle of the LED, the higher the received power of the photodiode. The half-power angle of $30^{\circ}$ was chosen and used when calculating the total received power for both the LOS and NLOS of $\mathrm{Li}-\mathrm{Fi}$. The total received power for both LOS and NLOS with a reflection is in fig. 8 

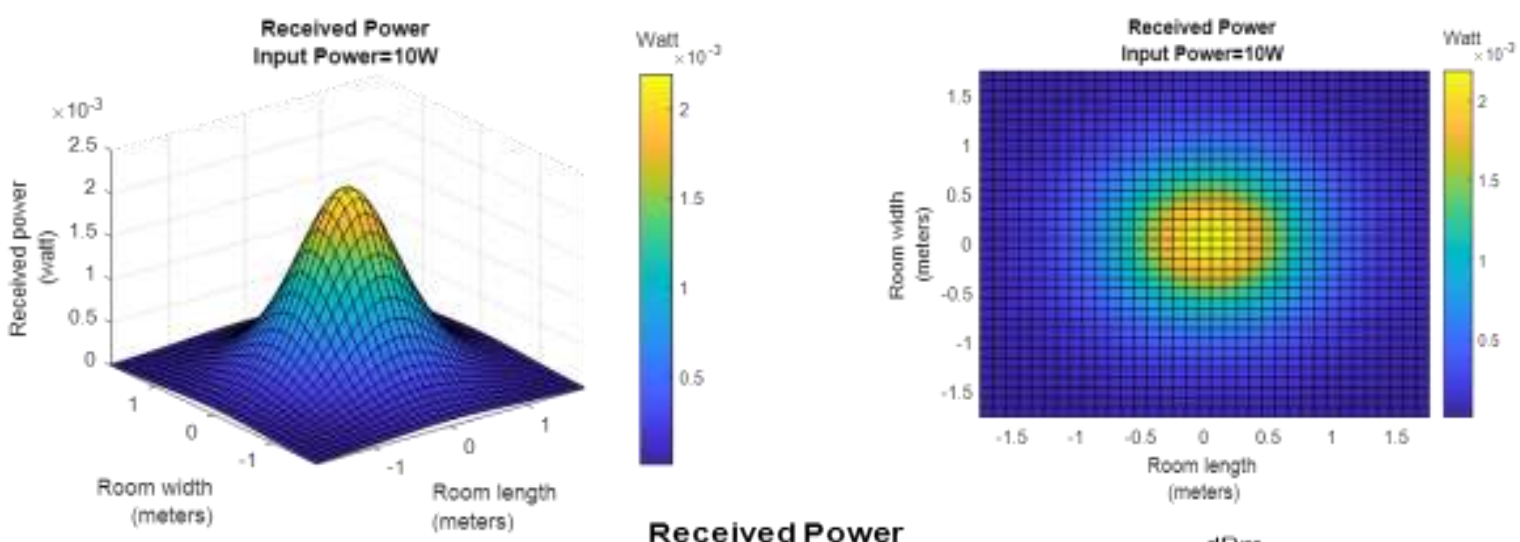

\section{Received Power} Input Power=10W

$\mathrm{dBm}$

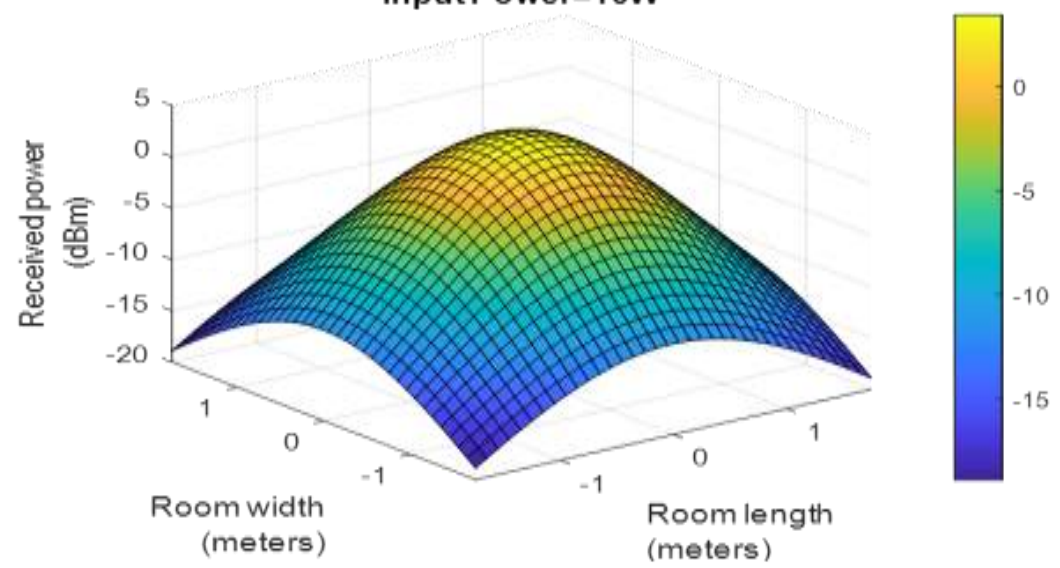

Fig. 6 Received power from photodiode at a half-power angle of $45^{\circ}$
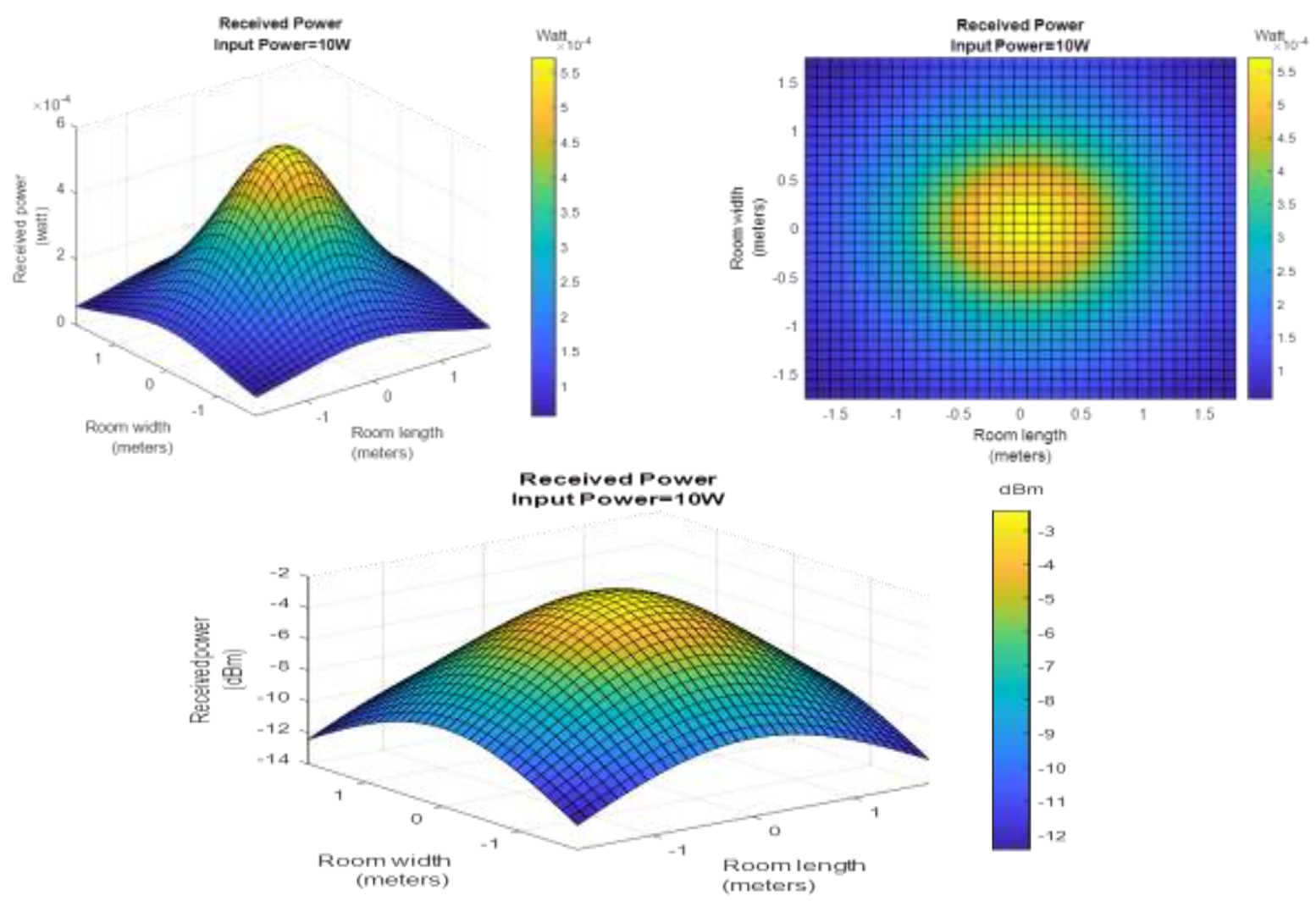

Fig. 7Received power from photodiode at a half-power angle of $60^{\circ}$ 


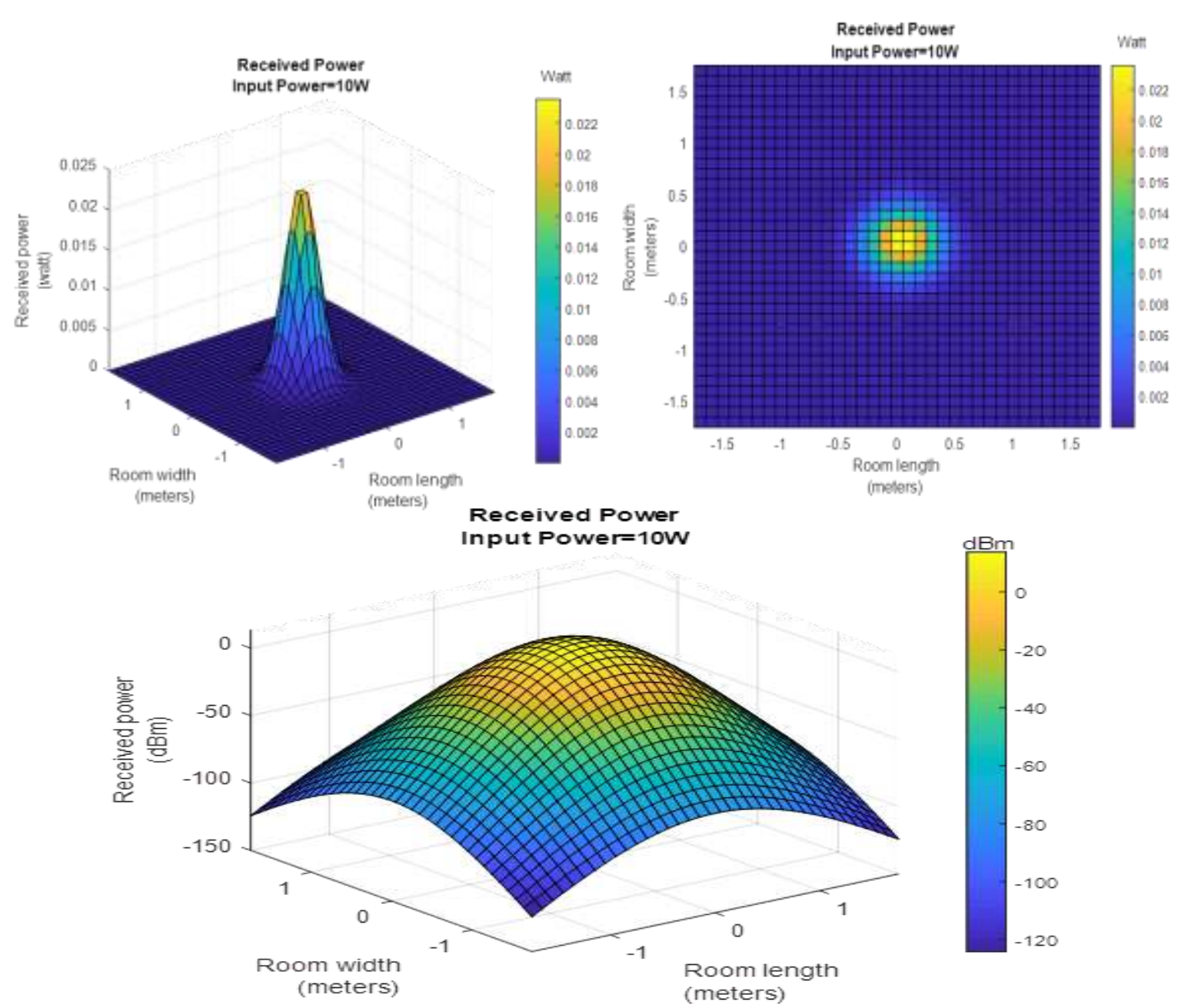

Fig. 8 Received power from photodiode for both LOS and NLOS

$\mathrm{Li}-\mathrm{Fi}$ at a half-power angle of $30^{\circ}$

It is the same with LOS obtained in fig.5 except that the received power increased and the range of values good for the reception is between $-60 \mathrm{dBm}$ to $0 \mathrm{dBm}$.

The result of the received power of Wi-Fi obtained using the two-ray ground reflection model is in fig. 9

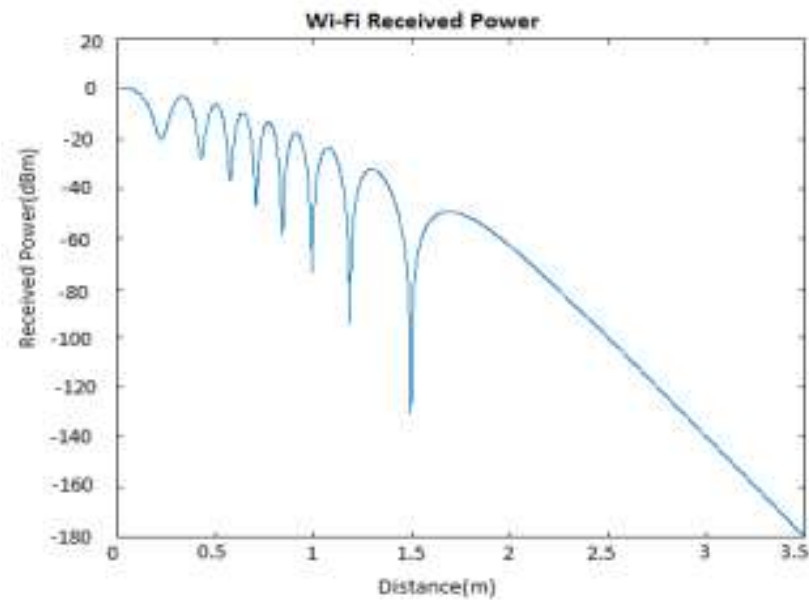

Fig. 9 Indoor wireless fidelityreceived power 
As the distance increases, the received power drops, giving regions of up fade and down fade, but the highest values for the reception is within the range of $-120 \mathrm{dBm}$ to $0 \mathrm{dBm}$.

Since the range of received power for good reception of $\mathrm{Li}-\mathrm{Fi}$ is from $-60 \mathrm{dBm}$ to $0 \mathrm{dBm}$, as shown in fig 4.28 and $\mathrm{Wi}-\mathrm{Fi}$ is $-120 \mathrm{dBm}$ to $0 \mathrm{dBm}$ as shown in fig 4.29 , the Wi-Fi signal is used only when the received power of Li-Fi system is less than $-60 \mathrm{dBm}$.

\section{CONClusion}

The hybrid networks of light fidelity and wireless fidelity are an emerging network for future indoor wireless communication. It can improve the system throughput, and users experience while accessing the internet. Future work needs an extension of it for both the indoor and outdoor environments.

\section{ACKNOWLEDGMENT}

The authors wish to thank Enugu State University of Science and TechnologyNigeria,for providing the Laboratory and equipment for the accomplishment of this research

\section{REFERENCES}

[1] Shailandra, K. (2013). An Overview of Technical Aspect for Wifi Network Technology.International Journal of Electronics and Computer Science, 1(1), 28-34.

[2] Shridhar, A.,Megan, B., Calvin, N. (2015). Visible Light Communication.Worcester Polytechnic Institute, pp.1-69.https://web.wpi.edu/Pubs/E-project/Available/E-project032615-203414/unrestricted/Visible_Light_Communication_MQP_Report_Final_20142015.pdf. Accessed on May 5, 2017.

[3] Bradley, K.(2016) Introduction to Wi-Fi Wireless Networking. LifeWire. https://www.lifewire.com/introduction-to-wi-fi-wireless-networking-818265, Accessed on: Aug. 2018.

[4] Haas, H. (2015). What is Li-Fi?Journal of Lightwave Technology, 34(6),1533-1544.

[5] Ashwini,B. N., Avikumar, R. (2016).Wireless Data Transmission Through LED.International Journal of Engineering Research in Electrical and Electronic Engineering, 2( 5),42-45.

[6] Ghassemlooy, Z., Popoola, W.,Rajbhandari, S. (2018). Optical Wireless Communication.CRC Press Taylor and Francis Group, pp. 113-150.

[7] Yang, Q.,Hsiao-Hwa, C.,Wei-Xiao, M. (2016). Channel Modeling for Visible Light Communications- A Survey.Wireless Communications and Mobile Computing,16, 20162034.

[8] Chein, C. (2002), Digital Radio Systems on a Chip: A Systems Approach. Kluwer Academic Publishers ebook ISBN: 0-306-46993-6.

[9] Loyka, Sergey, Kouki, Ammar, (2001). Using Two Ray Multipath Model for Microwave Link Budget Analysis," IEEE Antennas and Propagation Magazine,43(5), 31-36. 